\title{
Review of: "Emergence of two distinct SARS-CoV-2 Gamma variants and the rapid spread of P.1-like-II SARS-CoV-2 during the second wave of COVID-19 in Santa Catarina, Southern Brazil"
}

\author{
Ana Beatriz Gorini Veiga \\ 1 Universidade Federal de Ciências da Saúde de Porto Alegre
}

Potential competing interests: The author(s) declared that no potential competing interests exist.

In this study, samples from SARS-CoV-2-positive individuals from the state of Santa Catarina, in southern Brazil, were submitted to NGS for sequencing of the viral genome. The sequences were compared to other SARS-CoV-2 sequences from Santa Catarina and other geographic regions to identify viral variants, mutations, and dynamics. The study was well designed and very similar to other studies performed in Brazil, however in other states, therefore it adds important data for the genomic surveillance of SARS-CoV-2.

Considering that the state of Santa Catarina is a touristic destination and that, because of the pandemic, many people stopped traveling abroad and traveled mainly in the country, I think it would be interesting to discuss a possible impact of tourism and touristic activities (especially in the summer time) in the entrance and dispersion of SARS-CoV-2 variants. 